\title{
Creencias docentes acerca de la enseñanza de la argumentación en el ciclo básico de formación
}

\author{
Antonia Larraín ${ }^{1}$, Leandro De Brasi ${ }^{2}$, Maribel Calderón ${ }^{1}$ y Alejandro Calzetta ${ }^{3}$ \\ (1) Facultad de Psicología, Universidad Alberto Hurtado, Santiago, Chile (correo-e: alarrain@uahurtado.cl, \\ mcalderon@uahurtado.cl) \\ (2) Centro de Investigación de las Humanidades, Universidad de La Frontera, Temuco, Chile \\ (correo-e: I.debrasi@outlook.com) \\ (3) Facultad de Derecho, Universidad Alberto Hurtado, Santiago, Chile (correo-e: acalzetta@uahurtado.cl) \\ *Autor a quien debe ser dirigida la correspondencia
}

Recibido May. 22, 2020; Aceptado Jul. 17, 2020; Versión final Ago. 17, 2020, Publicado Feb. 2021

\begin{abstract}
Resumen
El objetivo de este estudio es describir las creencias de docentes y su rol en la enseñanza de habilidades de argumentación en la formación universitaria. Se realiza un estudio exploratorio-descriptivo con enfoque cualitativo, en el que participaron 16 docentes que enseñaron los primeros dos años de carreras de filosofía y derecho de una universidad privada chilena en el año 2019. Se realizaron entrevistas en profundidad realizando codificación temática. Los resultados muestran alta coincidencia en las creencias docentes: se valora la argumentación como competencia clave, pero se plantea que debe enseñarse una vez se asienten conocimientos teóricos. Si bien los docentes reportan promover prácticas de argumentación en su enseñanza, no lo hacen para desarrollar argumentación. La evaluación de aprendizaje supone saber argumentar, pero no se entregan pautas que orienten esta línea. Se concluye que los docentes de carreras de filosofía y derecho no difieren radicalmente en sus creencias acerca del valor de la argumentación para el ejercicio profesional.
\end{abstract}

\section{Professor beliefs regarding the teaching of argumentation in undergraduate education}

\begin{abstract}
The aim of the present research study is to describe professor beliefs and their role in the teaching of argumentative skills in higher education. An explorative-descriptive study with a qualitative approach is performed. The population surveyed is composed of 16 professors that taught the first two years of philosophy and law undergraduate degrees at a private Chilean university in 2019. Interviews are performed with thematic analysis. The results show high agreement between professors in their beliefs: argumentation is valued as a key competence, but it is suggested that argumentation should be taught once knowledge of the discipline is acquired. Although professors report to promote argumentative practices in their teachings, they do not do it to develop argumentative skills. Learning evaluations implies knowing how to argue, but no assessment argumentation guidelines are provided. It is concluded that surveyed philosophy and law professors do not differ radically in their beliefs about the value of argumentation in professional practice.
\end{abstract}

Keywords: teaching argumentation; professor beliefs; university pedagogy 


\section{INTRODUCCIÓN}

La argumentación puede ser entendida como práctica discursiva que involucra la producción, evaluación y revisión de razones a favor y en contra de un cierto punto de vista (Andrews, 2009; Bova, 2017; Larraín y Burrows, 2020; Leitão, 2000). Como tal, es una práctica que juega un rol central en nuestras vidas, tanto a nivel individual como colectivo. A nivel individual y desde un punto de vista epistémico, la práctica de la argumentación mediante la consideración de puntos de vista alternativos y sus fundamentos, nos permite aspirar individualmente a creer según nuestras mejores razones, tener éxito en nuestras acciones y/o comprender creencias, acciones o fenómenos. Aún más importante, a nivel colectivo, la argumentación es una práctica clave en las democracias contemporáneas (Chambers, 2003), en la medida que los ciudadanos deben articular sus diferencias para imaginar soluciones que permitan sostener la vida en común. De hecho, es un compromiso básico de la democracia moderna que los ciudadanos deben poder participar en actos de crítica, protesta, resistencia y desacuerdo. Es más, muchos de los derechos y libertades que asociamos a la democracia, como la libertad de expresión, de prensa y de asociación, están directamente relacionados a nuestra aspiración social a que las vidas colectivas de las personas estén dirigidas por las mejores razones, es decir, por la fuerza de los mejores argumentos (Habermas, 1996). Por lo tanto, una parte crucial de la democracia es el intento de argumentar uno con el otro acerca de lo que colectivamente, deberíamos hacer. De esta manera, las habilidades de argumentación son un objetivo estratégico para la vida en común.

Sin embargo, argumentar pertinentemente es algo que se aprende y que requiere acciones de enseñanza explícitas y deliberadas (Larraín y Burrows, 2020). Lo poco que sabemos acerca del impacto del sistema educativo en Chile en las habilidades de argumentación de estudiantes, sugiere que a nivel escolar no se provee de oportunidades sistemáticas para el desarrollo de habilidades de argumentación (Larraín et al., 2014) y que si bien estudiantes universitarios avanzan en su desarrollo (Larraín et al., 2015; Tuzinkievicz et al., 2018) no es claro si este avance se debe a oportunidades de aprendizaje explícitas y deliberadas. Considerando la importancia actual que tiene fortalecer el ejercicio de una ciudadanía deliberativa en jóvenes, la formación universitaria en Chile debiera asumir seriamente el compromiso de promover habilidades de argumentación, especialmente en aquellos que tendrán responsabilidades claves en la vida pública. Este artículo reporta un estudio que pretende contribuir a describir las creencias de docentes de carreras de Filosofía y Derecho de una universidad inclusiva de la Región Metropolitana acerca de el aprendizaje y la enseñanza de habilidades de argumentación. El estudio estuvo guiado por las siguientes preguntas: ¿Cuáles son las creencias de docentes universitarios de carreras de Filosofía y Derecho respecto a la formación de habilidades de argumentación y al rol de su enseñanza y prácticas pedagógicas en esta?

\section{OTROS ANTECEDENTES}

Hay una serie de antecedentes sobre el tema que es necesario detallar para documentar en mejor forma este trabajo. En particular asuntos sobre el ddesarrollo de habilidades de argumentación y la docencia y desarrollo de habilidades argumentativas.

\section{Desarrollo de habilidades de argumentación}

Existen diferentes aproximaciones teóricas respecto a qué es argumentar (Rapanta et al., 2013), estando más allá del presente trabajo abordarlas de manera comprensiva. En este texto, cuando hablamos de argumentación nos referimos a un modo específico de conversar o pensar que se caracteriza por ofrecer evidencia y justificación explícita al momento de sostener una posición, al tiempo de considerar objeciones y límites de esta, como parte de una actividad dialéctica en el que se confrontan perspectivas (Kuhn, 2019). La argumentación puede desarrollarse de manera oral o escrita, y aunque estos modos comparten características communes (justificación, articular conclusion y evidencia, anticipar y formular contraargumentos, enre otros) también imponen diferentes demandas cognitivas (Coirier et al., 1999). Mientras en la oralidad interlocutores co-construyen el proceso argumentativo, en la escritura el peso de la producción recae en un hablante quien debe anticipar la audiencia y su contexto, (Newell et al., 2011). Desde este punto de vista, comprendemos la argumentación es una práctica social que puede desarrollarse entre dos o más interlocutores, pero que también puede desarrollarse en el discurso de un mismo hablante (ver Greco, 2017), como es el caso de la escritura. Se sigue de lo anterior que la argumentación involucra no solo un proceso interpersonal, sino tiene un carácter dialógico (Leitão, 2000), involucrando la habilidad de usar el lenguaje estratégicamente para formular argumentos y contra-argumentos ante asuntos controversiales (Coirier et al., 1999); anticipar contra-argumentos y responderlos (Felton, 2004); y persuadir a interlocutores reales 0 anticipados para hacerlos o bien cambiar de opinión o lograr un entendimiento (Coirier et al., 1999). Llamaremos a esta habilidad de argumentación o argumentativa.

La literatura muestra que las habilidades de argumentación se desarrollan tanto en su estructura (argumento, contra-argumentos y refutaciones; ver Felton, 2004), complejidad (en tanto cantidad y relación entre premisas 
y conclusión; ver Stein y Albro, 2001) y uso estratégico (uso de acuerdo a contextos, situación y audiencia para el cumplimiento de objetivos; ver Felton y Kuhn, 2001). Si bien ya a los cuatro años de edad niños y niñas producen todas las partes relevantes de un argumento en la medida que crecen, van avanzando en su capacidad de producir y articular más razones para apoyar sus puntos de vista, formular más y más complejos contra-argumentos, e identificar las debilidades de los argumentos de sus oponentes (Stein y Albro, 2001). Sin embargo, la capacidad estratégica y de anticipar contra-argumentos a los argumentos propios y ajenos se estanca en la adolescencia (Felton y Kuhn, 2001; Stein y Albro, 2001), requiriendo esfuerzos explícitos de instrucción y enseñanza.

La evidencia disponible muestra que las habilidades argumentativas se desarrollan a través de la participación en prácticas argumentativas con cuidadores y profesores (McNaughton et al., 2019) y entre pares (Felton, 2004; Felton y Kuhn, 2001; Iordanou et al., 2019). Más aún, la evidencia muestra que la argumentación oral y escrita se potencia mutuamente (Chen et al., 2016) y que apoyos instruccionales directos específicos como pautas o andamios tienen efectos positivos en la escritura argumentativa de estudiantes universitarios (Larraín y Burrows, 2020). En síntesis, se puede enseñar a argumentar, ya sea diseñando interacciones que permitan practicar la argumentación oral de manera intensiva, u ofreciendo apoyos explícitos para el mejoramiento de las habilidades argumentativas argumentativas.

Ahora bien, enseñar a argumentar enfrenta dificultades. Por un lado, los criterios de calidad para construir y evaluar la calidad argumentativa (importante para saber si se ha promovido desarrollo de esta habilidad) cambian según disciplina y condiciones retóricas -objetivos y audiencia (Andrews, 2009; Newell et al., 2011). Por otro lado, según Rapanta et al. (2013) la literatura en educación enfatiza distintos niveles del proceso: el nivel metacognitivo (la estructura de argumentación, la precisión conceptual del tema argumentado y la calidad epistémica en la justificación entre premisas y su justificación); el nivel meta-estratégico (la competencia comunicativa, la comprensión del contexto de la tarea y la implementación de estrategias argumentativas de alto nivel); y, por último, el nivel epistémico (la relevancia, suficiencia y aceptabilidad del argumento). Así, la pregunta por qué aspecto focalizar al momento de enseñar a argumentar puede ser un factor de incertidumbre para la enseñanza de argumentación.

Por otro lado, la promoción de habilidades de argumentación en contextos de enseñanza formal involucra otro aspecto: el conocimiento. Típicamente, por ejemplo, en la escuela o en carreras universitarias, además del desarrollo de habilidades, el objetivo de enseñanza involucra la apropiación de conocimiento. Una alternativa es promover el desarrollo de conocimiento y de argumentación (aprender a argumentar) como dos objetivos de aprendizaje independientes, que rivalizarán en términos de objetivos formativos. Más aún, se puede pensar que para argumentar se requiere conocimiento pues hay evidencia que muestra que la familiaridad del contenido es un factor relevante para el desempeño argumentativo (Coirier et al., 1999). Sin embargo, la literatura muestra que la argumentación es un medio privilegiado para construir conocimiento y que cuando estudiantes universitarios argumentan, comprenden mejor conceptos propios de las disciplinas formales (Asterhan y Schwarz, 2016). Esto implicaría que aprender a argumentar y argumentar para aprender serían dos objetivos interdependientes que pueden ser logrados en una misma intervención pedagógica (lordanou et al., 2019) y que se potenciarían mutuamente.

\section{Docencia y desarrollo de habilidades argumentativas}

Las prácticas pedagógicas que promueven el desarrollo de habilidades argumentativas en el marco de la enseñanza de contenidos disciplinares tales como argumentación oral entre pares y docentes, 0 argumentación escrita colaborativa o andamiada, guiada y retroalimentada, son poco frecuentes en la enseñanza regular (McNeill et al., 2016). En la docencia universitaria si bien la argumentación está presente en muchas de las herramientas para evaluar aprendizaje universitario (i.e. ensayo argumentativo), las habilidades de argumentación se tienden a suponer más que a enseñar (ver Andrews, 2009). Esto es problemático pues se evalúan aspectos que no se enseñan explícitamente, lo que suele afectar el desempeño de los estudiantes. La enseñanza de habilidades argumentativas es una tarea difícil y se ha reportado a nivel universitario, que docentes no saben cómo enseñar a argumentar conforme a normas propias de la disciplina e incluso no logran explicitar los criterios de calidad (Newell et al., 2011). De hecho, Andrews (2009) señala que los docentes típicamente abordan la argumentación superficialmente y asumen que los estudiantes ya manejan la competencia. Además, los docentes tienden a no contar con formación en argumentación y por lo tanto desconocen las capacidades específicas que necesitan desarrollar en sus estudiantes. Un estudio reportado en Andrews (2009) muestra diferencias disciplinares interesantes: estudiantes de biología e ingeniería presentaban mayores dificultades, en comparación de estudiantes de historia, para argumentar y para leer argumentativamente textos científicos.

Lo poco que sabemos respecto a la enseñanza de la argumentación en la carrera de Derecho, sugiere que las habilidades de argumentación tienen poco espacio (Robbins-Tiscione, 2006). La necesidad de promover 
la argumentación legal escrita sería clave pero altamente demandante para docentes universitarios (RobbinsTiscione, 2006). De acuerdo a Blasi (1999) existen intentos en las escuelas de Derecho de promover habilidades argumentativas pero no son efectivos. Las salas de clase numerosas, los currículos prolongados y las evaluaciones que demandan elaborar argumentos bajo presión, sin desarrollo o evaluación crítica, mermarían la posibilidad de una enseñanza efectiva en este ámbito. Sin embargo, la discusión en aula de argumentos escritos o sistemas computacionales para el desarrollo de argumentación legal (Pinkwart et al., 2006) han mostrado ser vías posibles.

Respecto a Filosofía, lliadi et al. (2019) muestran que estudiantes de esta carrera, y en mayor medida los de cursos avanzados, desarrollan más y mejores habilidades argumentativas, en comparación con quienes no han cursado Filosofía. Sin embargo, aún estos estudiantes no tendrían plena fluidez en conceptos básicos de argumentación ni en la evaluación de argumentos, lo que enfatiza la importancia de la enseñanza explícita e intencionada. Las habilidades argumentativas serían particularmente claves en el aprendizaje de la escritura de ensayos filosóficos, lo que representaría una dificultad importante para estudiantes (Martinich, 2016). Se enfatiza la necesidad de guiar explícitamente a estudiantes en la escritura de estos ensayos. De hecho, un estudio muestra mejoras en la capacidad de estudiantes de analizar argumentos al representarlos visualmente mediante esquemas, lo que sería especialmente efectivos en estudiantes de desempeños iniciales más bajos (Harrell, 2011).

La escasez de prácticas pedagógicas orientadas a promover las habilidades de argumentación se atribuye, en parte, a la falta de un contexto retórico propicio para argumentar en las aulas regulares (ver Coirier et al., 1999). Por ejemplo, a la asimetría de poder existente entre docentes y estudiantes; a la falta de contenidos polémicos o controversiales; a que el resultado de la argumentación en la enseñanza disciplinar, especialmente escolar, no está abierto sino predefinido; a que los estudiantes no cuentan con suficiente familiaridad con el tema, entre otros aspectos. Sin embargo, las creencias docentes también se consideran como obstáculos para la enseñanza de la argumentación. Aunque la relación unidireccional de las creencias como determinando las prácticas está hoy puesta en cuestión (Martin y Dismuke, 2018), es indudable que estas participan de manera relevante de la enseñanza, ya sea expresando el conocimiento práctico docente como influyendo en la práctica pedagógica misma.

Respecto de las creencias acerca de la enseñanza de argumentación de docentes, sabemos extremadamente poco. Uno de los pocos estudios que estudian creencias docentes respecto a la enseñanza de la argumentación (McNeill et al., 2016) muestra que si bien docentes escolares creen que la enseñanza de la argumentación es importante, no existía claridad de por qué en particular esto es así. Además, si bien creen que todos los estudiantes pueden aprender a argumentar, las habilidades de estudiantes sí impactan en su enseñanza. Por último, el estudio muestra que si bien la mayoría de docentes se sentía confiado de sus habilidades para enseñar a argumentar, eso dependía de su experiencia en esto y del conocimiento de sus estudiantes. En términos de creencias docentes en la enseñanza universitaria, se ha reportado (Andrews, 2009) que los docentes estipulan que la argumentación no es su responsabilidad y que los estudiantes la aprenderán durante la carrera, acaso con el apoyo de otra entidad (por ejemplo, de los centros de escritura). Más aún, los docentes de biología e ingeniería creen que el foco de los primeros años es desarrollar comprensión del campo y conceptos claves, para argumentar con ellos hacia el cierre de la carrera o en estudios de posgrado. Esto contrasta con el caso de historia, en la que estudiantes y académicos subrayan la centralidad de la argumentación, generando instancias para cuestionar críticamente fuentes terciarias 0 secundarias desde el inicio del pregrado.

De esta manera, poco se sabe de las prácticas concretas con que docentes universitarios enseñan a argumentar y de las creencias de docentes respecto a esto. Existen estudios que evalúan eficacia de aspectos instruccionales como uso de pautas (Larraín y Burrows, 2020), análisis visual de argumentos (Harrell, 2011) y uso de andamios computacionales (Pinkwart et al., 2006), pero se sabe poco de lo que realmente se hace en educación universitaria para enseñar a argumentar, menos aún en carreras que tienen un impacto tan importante en la vida pública como Filosofía y Derecho. Estas carreras típicamente incluyen en sus perfiles de egreso la argumentación como una competencia clave, pero la pregunta es cómo docentes que enseñan en estas carreras entienden su rol formativo en esta línea. Con este propósito llevamos a cabo un estudio que describe las creencias de docentes de carreras de Filosofía y Derecho respecto a la formación de habilidades de argumentación en la enseñanza universitaria y al rol de su enseñanza y prácticas pedagógicas en esta.

\section{METODOLOGÍA}

El diseño del estudio fue exploratorio-descriptivo con enfoque cualitativo, utilizando la entrevista en profundidad semi-estructurada como técnica de producción de información. Los participantes del estudio fueron 16 docentes que enseñan en una universidad privada de la ciudad de Santiago de Chile. Participaron 8 docentes de la carrera de Filosofía y 8 de Derecho (14 hombres y 2 mujeres-una de cada carrera). Entre 
ellos había 12 docentes con contrato de planta (6 de Filosofía) y 4 docentes con contrato por hora (2 de Filosofía) del Ciclo Básico.

De una lista de la totalidad de docentes que hacen clases en el Ciclo Básico (esto es, los dos primeros años) de las Carreras de Filosofía y Derecho de una universidad privada chilena, se invitó a participar a los y las participantes. Cuatro psicólogos (tres mujeres) coordinaron y realizaron las entrevistas (una por docente), las que se realizaron en dependencias de la Universidad, teniendo una duración promedio de 78 minutos con un mínimo de 38 y un máximo de 114). Estas se grabaron en audio. El trabajo de campo se realizó entre junio y agosto de 2019. Al momento de la entrevista y antes de comenzar, cada académico firmó un consentimiento informado donde se describían las condiciones de participación y alcance del proyecto.

Las entrevistas estuvieron orientadas por un guion temático para indagar las opiniones docentes respecto a: 1) en general, de la argumentación, su valor profesional y desarrollo; 2) respecto a sus propias prácticas tendientes a promover habilidades argumentativas. Así, se incluyeron los siguientes ámbitos rol de la argumentación en el ejercicio profesional y la formación; desarrollo de habilidades argumentativas; prácticas de enseñanza para el fomento de las habilidades de argumentación; prácticas de evaluación del aprendizaje para el desarrollo de habilidades de argumentación; y dificultades y facilitadores. El guion fue construido iterativamente en a partir de una revisión bibliográfica. Una primera propuesta levantada por una de las autoras en base a la literatura fue revisada y comentada por el resto de investigadores del proyecto. Las preguntas fueron utilizadas en una entrevista piloto y ajustada para las siguientes entrevistas. El análisis se realizó mediante codificación temática por categorías iniciales que se presentan en Tabla 1, siendo revisada por dos analistas cada una. Luego, se llevaron a cabo rondas de revisión de categorías pre-establecidas como mecanismo de validación intersubjetiva por cinco miembros del equipo de investigación.

Tabla 1. Categoría de análisis temático

\begin{tabular}{|c|c|}
\hline Categorías iniciales & Descripción \\
\hline Noción de argumentación & Concepciones, ideas o nociones de la argumentación en la disciplina. \\
\hline $\begin{array}{l}\text { Formación docente en relación con la } \\
\text { argumentación }\end{array}$ & Habilidades y falencias personales para usar y enseñar a argumentar. \\
\hline $\begin{array}{l}\text { Argumentación en la formación de la } \\
\text { carrera y el quehacer profesional }\end{array}$ & $\begin{array}{l}\text { Valoraciones, justificaciones y ejemplos de la importancia de la } \\
\text { argumentación en la formación y la profesión. }\end{array}$ \\
\hline $\begin{array}{l}\text { Aporte curso al desarrollo de las } \\
\text { habilidades de argumentación }\end{array}$ & $\begin{array}{l}\text { Valoración y contribución del curso que se enseña en torno a habilidades } \\
\text { argumentativas. }\end{array}$ \\
\hline $\begin{array}{l}\text { Aspectos generales sobre argumentación } \\
\text { en cursos dictados }\end{array}$ & $\begin{array}{l}\text { Vinculación de las características del curso dictado con las habilidades } \\
\text { y/o prácticas argumentativas. }\end{array}$ \\
\hline $\begin{array}{l}\text { Características de los estudiantes de los } \\
\text { cursos en relación con la argumentación }\end{array}$ & $\begin{array}{l}\text { Concepciones sobre las características de los estudiantes que pueden } \\
\text { facilitar o limitar el trabajo argumentativo en el aula y el desarrollo de } \\
\text { competencias. }\end{array}$ \\
\hline $\begin{array}{l}\text { Prácticas pedagógicas en el aula: } \\
\text { instancias expositivas }\end{array}$ & $\begin{array}{l}\text { Uso de estrategias, herramientas o actividades expositivas en torno a la } \\
\text { argumentación. }\end{array}$ \\
\hline $\begin{array}{l}\text { Prácticas pedagógicas en el aula: } \\
\text { instancias activas }\end{array}$ & $\begin{array}{l}\text { Uso de estrategias, herramientas o actividades activas en torno a la } \\
\text { argumentación. }\end{array}$ \\
\hline $\begin{array}{l}\text { Prácticas pedagógicas en el aula: } \\
\text { ayudantias o asesorías }\end{array}$ & $\begin{array}{l}\text { Uso de estrategias, herramientas o actividades en ayudantías o } \\
\text { asesorías en torno a la argumentación }\end{array}$ \\
\hline $\begin{array}{l}\text { Prácticas pedagógicas: evaluación y } \\
\text { retroalimentación }\end{array}$ & $\begin{array}{l}\text { Uso de estrategias, herramientas o actividades de evaluación y } \\
\text { retroalimentación en torno a la argumentación. }\end{array}$ \\
\hline Gestión institucional & $\begin{array}{l}\text { Contribución de la gestión institucional al desarrollo de prácticas } \\
\text { argumentativas en la formación. }\end{array}$ \\
\hline $\begin{array}{l}\text { Instancias formales e informales de la } \\
\text { carrera }\end{array}$ & $\begin{array}{l}\text { Contribución formal e informal de la institución al desarrollo de prácticas } \\
\text { argumentativas en la formación. }\end{array}$ \\
\hline Orientaciones para la mejora & $\begin{array}{l}\text { Ideas respecto a mecanismos o herramientas de mejora para el } \\
\text { desarrollo de habilidades argumentativas }\end{array}$ \\
\hline
\end{tabular}

\section{RESULTADOS}

Para facilitar la exposición de los resultados, estos se presentan en varias subsecciones de acuerdo a las categorías resultantes (ver Tabla 2): argumentación, el aprendizaje de la argumentación, la enseñanza de la argumentación, argumentación en clases, evaluación de habilidades de argumentación y principal obstáculo formativo. 
Tabla 2. Relación entre categorías iniciales y categorías resultantes

\begin{tabular}{|c|c|c|}
\hline Categoría iniciales & $\rightarrow$ & Categorías resultantes \\
\hline Noción de argumentación & $\rightarrow$ & Argumentación \\
\hline Formación docente en relación con la argumentación & $\rightarrow$ & Aprendizaje de la argumentación \\
\hline $\begin{array}{l}\text { Argumentación en la formación de la carrera y el quehacer } \\
\text { profesional }\end{array}$ & \multirow[t]{2}{*}{$\rightarrow$} & \multirow[t]{2}{*}{ Enseñanza de la argumentación } \\
\hline $\begin{array}{l}\text { Aporte curso al desarrollo de las habilidades de } \\
\text { argumentación }\end{array}$ & & \\
\hline $\begin{array}{l}\text { Prácticas pedagógicas en el aula: instancias expositivas, } \\
\text { activas y asesorias. }\end{array}$ & $\rightarrow$ & Argumentación en clases \\
\hline Prácticas pedagógicas: evaluación y retroalimentación & \multirow[t]{3}{*}{$\rightarrow$} & \multirow[t]{3}{*}{ Evaluación de habilidades de argumentación } \\
\hline Instancias formales e informales de la carrera & & \\
\hline $\begin{array}{l}\text { Aspectos generales sobre argumentación en cursos } \\
\text { dictados }\end{array}$ & & \\
\hline $\begin{array}{l}\text { Características de los estudiantes de los cursos en relación } \\
\text { con la argumentación }\end{array}$ & \multirow[t]{3}{*}{$\rightarrow$} & \multirow[t]{3}{*}{ Principal obstáculo formativo } \\
\hline Gestión institucional & & \\
\hline Orientaciones para la mejora & & \\
\hline
\end{tabular}

\section{Argumentación}

Los y las docentes participantes acuerdan que la argumentación es una competencia clave para el ejercicio profesional. Sin embargo, la noción de argumentación y, con esto, la competencia requerida, difiere en ambas carreras. En Derecho aparecen dos aspectos de la argumentación igualmente relevantes. Por un lado, se trataría de una forma de razonamiento normativo que poseería una estructura (silogismo judicial), y un tipo de discurso que se reconoce en un marco interpretativo que debe ser aplicado de manera clara: "Una justificación interna, con argumentos deductivo-lógicos, donde existe una norma $A$ y hechos $B$, y la deducción es que se aplica la norma A a los hechos B" (Caso 8, Derecho).

Por otro lado, en la práctica profesional emergería como un proceso discursivo de carácter retórico que se centra en la solución de problemas: "Dar razones de cualquier tipo sobre la conveniencia o inconveniencia de tomar una decisión en un contexto de certeza, de riesgo o incertidumbre [...] el uso de argumentos está pensado para defender los intereses del cliente... Se debe convencer a otros de tomar ciertos cursos de acción que son jurídicamente más deseables" (Caso 2, Derecho). En el caso de Filosofía, aparece la idea de que la argumentación en tanto razonamiento bueno es clave para la práctica profesional: "[La argumentación es una] habilidad integradora de otras cosas: de la lectura, la fundamentación, del diálogo oral, de la consciencia de fundamentos de uno y de otros, y la capacidad de comprender posiciones no filosóficas [...] la habilidad, la capacidad, la competencia [...] de justificar, hilar lógico-racionalmente, afirmaciones, tesis, y en el contexto de un diálogo con otros, sea presencial o por texto" (Caso 16, Filosofía).

Se plantean aspectos vinculados a la claridad y capacidad de articular el pensamiento para tales fines: "un filósofo es, en cierta medida, una persona que habla y escribe bien, teniendo una comprensión lo más plural posible de la situación humana, de la sociedad y busca establecer los vínculos entre distintas instituciones que componen la sociedad para la comprensión del presente en toda su complejidad" (Caso 14, Filosofía). De esta manera, mientras en Derecho la argumentación aparece ligada al razonamiento normativo (carácter lógico) y persuasión (carácter retórico), en Filosofía aparece ligada a la construcción y reconstrucción de argumentos en tanto medio de construcción de conocimiento (carácter epistémico).

\section{Aprendizaje de la argumentación}

Los docentes tanto de Filosofía y Derecho coinciden en señalar que la formación de pregrado propia si bien implicaba el uso de argumentación al escribir ensayos y textos, no incluía retroalimentación y acompañamiento para su aprendizaje. De esta manera, perciben que aprendieron a argumentar en la práctica a través de la imitación y el ensayo y error, fundamentalmente en experiencias de postgrado: "En la medida que [uno] ve cómo otro construye argumentos, va aprendiendo a cómo construir los propios. Es necesaria mucha práctica, el ensayo y error, además de un buen nivel de lectura para poder construir las frases y escritos bien" (Caso 5, Derecho). "La principal forma de aprendizaje reside en la imitación, comprendiendo a través de la experiencia, hacer aquello que otros han hecho, guía la incorporación de metodologías y saberes [...] la imitación es lo que más funciona, lo esencial es imitar [...]" (Caso 12, Filosofía). Es así como reconocen que dada la manera de aprenderla perciben dificultad en saber cómo enseñarla: "Cuando uno es profesor, por osmosis sabe las cosas; el problema es transmitir esos conocimientos a los alumnos que aprendieron de distintas maneras" (Caso 5, Derecho). 


\section{Enseñar a argumentar}

Docentes de ambas carreras señalan que la argumentación debiera enseñarse en los últimos años de formación y que en el Ciclo Básico se requiere reforzar primero habilidades básicas. En ese contexto, el desarrollo de habilidades argumentativas, si bien considerado central, sería una habilidad que se trabajaría de manera solo indirecta o implícita en los cursos del Ciclo Básico. En esta etapa sería más importante que los estudiantes aprendieran primero ciertos contenidos teóricos antes de comenzar a producir argumentos: "Lo que busca es trasmitir conocimientos a los alumnos, de tal manera que esos alumnos adquieran cierto conocimiento del Derecho y la evaluación posterior, se hace respecto a su mayor o menor adquisición de conocimiento, más que determinar su capacidad de argumentar" (Caso 3, Derecho). "No me entusiasma la idea de hacer aparecer el problema sin el contenido. En la medida que el énfasis está en el texto, en la hermenéutica del texto, la argumentación desnuda (falacia, inconsistencias lógicas), no me parece que se justifique o se pueda trabajar desde la pura teoría" (Caso 10, Filosofía).

En Filosofía, se enfatiza, además, la importancia de comprender y reconstruir argumentos ajenos primero, como foco del Ciclo Básico, para luego producir los propios: "Tratar de enseñarle a los estudiantes a reconocer argumentos, a ver cómo se construye un argumento, a ver cómo un autor defiende sus ideas, a ver cómo un autor recoge de otros autores las opiniones que le sirven a él para elaborar su propia opinión" (Caso 14, Filosofía).

\section{Argumentación en clases}

En general, se reporta una variedad de prácticas pedagógicas en las que si bien se promueven interacciones argumentativas estas no aparecen como objetivos de aprendizaje en sí mismos, sino más bien como medio para el logro de objetivos de aprendizaje ligado al manejo de teorías y conceptos. "El recurso a los ejemplos, a las preguntas a los estudiantes, a recoger ideas contrapuestas, de que justifiquen, si bien no estoy pensando en la argumentación, sí que sea un medio de que pueda percibir si han comprendido la idea que se trata de transmitir o no [...] Lo que uno transmite son síntesis de ideas de otros autores o conceptos decantados [...] Que sean capaces de identificar situaciones en la misma lógica, más que formular argumentos propios" (Caso 2, Derecho).

La mayor parte de los docentes declara que las interacciones en ambas carreras se organizan en clase completa. Si bien en Derecho predomina la lógica de presentación de contenidos seguido de una parte más activa (preguntas y respuestas, trabajo de aplicación individual o grupal), en Filosofía predomina la clase magistral siguiendo la tradición del método socrático: "Por ejemplo, un párrafo del Laques de Platón, o algún tema importante, hago una comparación, muchas veces pongo el griego, el transliterado, para que ellos se conecten más con el texto original pero no les doy resuelto el texto, los expongo, lo leemos juntos, ya esto dijo Platón, pero yo soy la mediadora [...] Mi trabajo es de mediación lectora... Este es un lugar clásico, por qué se habla de la incontinencia, fíjense en las palabras que usa Sócrates, como le responde Laques, las ironías, qué palabras tienen más sentido" (Caso 15, Filosofía).

En Derecho, las interacciones argumentativas aparecen justificadas desde el punto de vista de promover el aprendizaje activo y la participación de estudiantes. Por ejemplo, un docente refiere al uso de "juegos de interpretación de fuentes" donde el profesor "juega" defendiendo una interpretación que sabe que está mal interpretada jurídicamente, pero lo hace para que ellos intenten desafiar esa idea y defender su postura propia (generalmente progresista), pero no de manera política, sino que de manera jurídica. Cuando se refiere a porqué se hace, se refiere a que serviría para que hablen y para que aprender sea más atractivo y no necesariamente para promover argumentación (Caso 1, Derecho).

Algunos docentes, especialmente en Derecho, valoran el trabajo entre pares pues la discusión permitiría la construcción organizada de argumentos y la toma de posición, que posibilitan identificar las propias habilidades y falencias: "No imagino el trabajo argumentativo en forma individualista, porque parte del resultado de lo que se espera de la argumentación, supone identificar las debilidades de la propia argumentación y las fortalezas de la argumentación contraria" (Caso 2, Derecho).

Solo dos docentes declararon el uso de generación de controversias o contra-argumentos durante sus clases. De hecho, estas se conciben como un espacio de oportunidad que no es frecuente, y no se promueve intencionalmente, pero que se aprovecha cuando ocurre: "Esos son momentos vitales de la clase, cuando hay una controversia, hay más de una opinión sobre algo y opiniones que están enfrentadas entre si [...] Un momento de participación activa, en el que salen de su letargo permanente. Sobre todo de los que están participando de la controversia, eso genera una suerte de protagonismo, la necesidad de ser contundentes, sólidos en la argumentación, de ser capaces de persuadir" (Caso 10, Filosofía). En ambas carreras, las estrategias que implícitamente promueven habilidades de argumentación se centran en la argumentación 
oral y en menor medida la argumentación escrita. Se pondría énfasis en la comprensión de argumentos, en la producción y finalmente en la evaluación.

\section{Evaluación de las habilidades de argumentación}

En ambas carreras no se reporta una clara conciencia de evaluar habilidades argumentativas: "Pero es secundario [lo argumentativo jurídico] [...] porque el objetivo primario como docente es otro: que maneje conceptos, que sepa aplicarlos, que sepa reconocerlos. Y con eso más o menos el tipo tiene un esquema general y después aprenderá argumentación y verá casos, y todo lo demás, pero si el tipo no sabe lo que es una fuente formal del Derecho, precariamente va a entender una sentencia del Tribunal Constitucional" (Caso 1, Derecho). Sin embargo, se reporta hacer en concreto evaluaciones que suponen habilidades argumentativas: "Deben ser capaces de establecer relaciones a partir del texto con los contenidos, construir una respuesta que no es univoca, si no que una concatenación entre distintos aspectos que hay que unificar o volver coherente. La respuesta es un argumento, no la descripción de un concepto" (Caso 10, Filosofía).

Más aún, en general no se reporta uso de pautas y criterios de evaluación específicos para esta dimensión, salvo una excepción en Filosofía donde se expone un caso de trabajo conjunto con otra asignatura del curso de Expresión Escrita, y donde se delega la revisión de los aspectos argumentales a ese curso. En Filosofía dos docentes indican que la rúbrica sería algo así como "una camisa de fuerza" que tendería a la uniformización y cierta rigidez para evaluar, razón por la cual no la usarían con sus estudiantes: "[La rúbrica] le pone una camisa de fuerza a la evaluación y prefiero ser más libre al momento de evaluar, en la medida en que he declarado de lo que voy a evaluar" (Caso 10, Filosofía)

Respecto a la retroalimentación, no se especifica retroalimentación focalizada en argumentación en ninguna de las dos carreras, estando más dirigida hacia aspectos conceptuales. Mientras docentes de Derecho plantean que las retroalimentaciones son acotadas por la cantidad de estudiantes por clase y que se hace principalmente de manera oral en clases, en Filosofía se reporta hacerlo mediante retroalimentaciones individuales por escrito.

\section{Principal obstáculo formativo}

Tanto docentes de Filosofía como de Derecho están de acuerdo que los principales obstaculizadores del proceso de enseñanza y aprendizaje son las habilidades de base de estudiantes, vinculados al capital cultural, cuya carencia dificultan el desarrollo esperado: "Uno se encuentra con problemas más básicos de estructuración gramatical, ortografía, o de comprensión de términos de lenguaje natural, mal uso semántico de ciertas expresiones" (Caso 13, Filosofía).

Estas carencias también limitarían a estudiantes a expresar sus puntos de vista por aprehensiones o desconocimiento del contexto donde se sitúan. Se menciona que no se espera que los estudiantes lleguen a la clase con habilidades particulares, si no que se encuentren con el desafío de generar destrezas específicas, como la rigurosidad en la lectura e interpretación de textos, así como la construcción y composición de sus propios trabajos: "No sé si las necesite [habilidades argumentativas] en el primer año [...] Pero sí podría haber algo del capital cultural... que tendría que ver con sus experiencias de lecturas que pueden hacer los estudiantes... Ya sean científicas o literarias" (Caso 12, Filosofía).

Otras dificultades se relacionan a la gestión pedagógica. Los académicos señalan que resulta muy difícil un trabajo más personalizado o de mayor monitoreo con la cantidad de estudiantes por sección y el tiempo disponible: "Es una cosa bien demencial, si uno también tiene capacidades físicas limitadas (...) y obviamente es un desgaste, porque los divides en grupos de a 8 o de a 6 , y te preguntan exactamente lo mismo diez veces [...] Eso lo puedes hacer una vez al semestre, dos veces al semestre. Pero también tienes que pasar materia" (Caso 1, Derecho). Lo anterior se vincula además con la percepción de tener pocas competencias o habilidades pedagógicas para enseñar a esos estudiantes, aplicando "metodologías intuitivas" en sus cursos. "Sabemos poco de cómo se aprende [...] No necesariamente sabemos comunicarlo a la medida del que lo tiene que recibir. A veces le pedimos cosas que no corresponde que las sepan. O que son habilidades que tienen en la habilidad oral, pero no en la expresión escrita" (Caso 11, Filosofía).

Docentes de Derecho señalan la necesidad de pensar la gestión institucional, para tener un posicionamiento y un marco claro y compartido hacia dónde dirigir los esfuerzos: "No sé si hemos conversado sobre el tipo de alumnos que tenemos, y creo que ese es el primer paso antes de cualquier estudio de cualquier cosa. Es el piso mínimo, qué tengo y cómo lo tengo. Y de ahí para adelante, si no, vamos inventando cursos de Lógica y Argumentación jurídica, electivos, y cosas súper choras. Pero ¿a quién le estás dando este producto? Yo creo que ahí hay que sincerar un poco sobre cuál es el tipo de alumno que tenemos y con qué dificultades" (Caso 1, Derecho). 
En esa misma línea, se señala que los profesores debieran compartir más entre ellos, pues existen docentes que tienen "metodologías intuitivas súper buenas"; sin embargo, las reuniones que existen son poco frecuentes, agregando a esto una dificultad de modo de ser de la cultura disciplinar que no favorece el trabajo conjunto. En el caso de Filosofía, si bien se reporta un mayor trabajo pedagógico conjunto entre pares, dado que la Filosofía está estrechamente vinculada a la argumentación se debiese hacer una reflexión mayor respecto a ella: "Debe haber un énfasis fundamental en argumentación, porque eso hacemos al filosofar. Un examen más profundo de los presupuestos de esa argumentación, una preocupación por hacernos entender y comunicar ideas" (Caso 10, Filosofía). Se plantea la necesidad de que la Universidad ofrezca espacios de formación formales en "modelos de trabajo con argumentación adaptable a los distintos momentos de los cursos"; es decir, que considere un aspecto teórico, pero por sobre todo, formas de aplicación y específicas según distintos niveles.

En síntesis, como se observa en la Figura 1, es posible organizar los resultados en tres grandes dimensiones a través de las que se organizan las creencias de docentes de las Carreras de derecho y Filosofía: creencias acerca de la argumentación, su aprendizaje y desarrollo; acerca de su propio rol y práctica pedagógica en la formación de estas habilidades; y acerca de obstáculos o barreras para su formación. Respecto a la primera dimensión, destaca el hecho de que si bien la argumentación se considera una competencia clave para el desarrollo profesional, se comparte la creencia que estas se aprenden de manera incidental en experiencias posteriores al ciclo básico pues se necesitarían bases conceptuales de la disciplina para poder desplegarlas. Así, y lo que destaca en la segunda dimensión, los y las docentes no visualizan la argumentación como objetivo formativo en sus cursos, lo que es coherente con la falta de reporte de estrategias sistemáticas y deliberadas para su desarrollo y enseñanza. Sin embargo, se reportan prácticas de promoción de argumentación que para los y las docentes están ligadas a la noción de aprendizaje activo y su importancia. Por último, respecto a la tercera dimensión los y las docentes creen que las habilidades de base de estudiantes son el principal obstáculo para la enseñanza de la argumentación, junto con un excesivo número de estudiantes en aula, y la falta de orientaciones y formación acerca de cómo hacerlo.

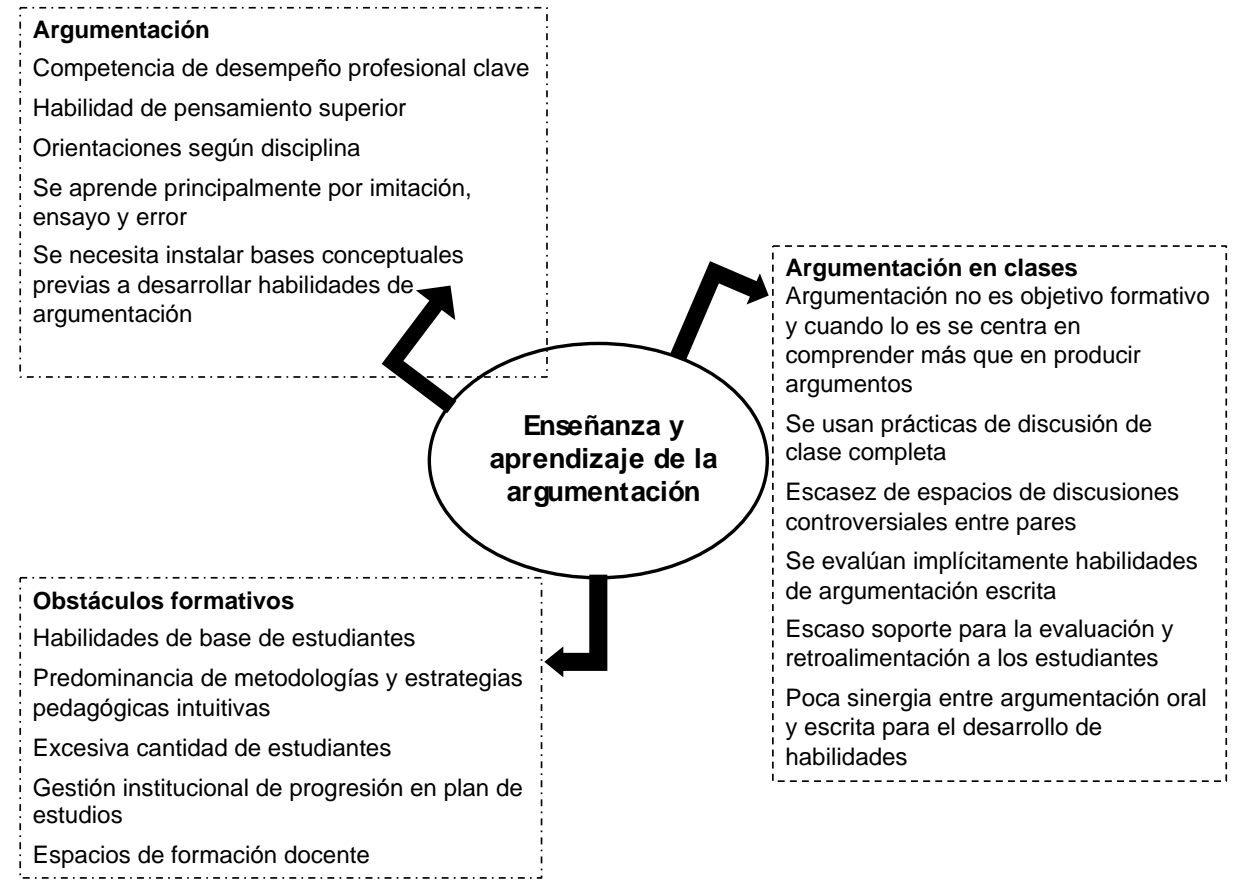

Fig. 1. Dimensiones que organizan las creencias docentes en torno a la enseñanza de la argumentación

\section{DISCUSIÓN}

En síntesis, existen importantes coincidencias entre docentes de Filosofía y Derecho, sugiriendo que más que un trabajo específico de cada carrera, las creencias respecto a la argumentación de docentes universitarios dependen en mayor medida de creencias y supuestos previos y compartidos que no se relacionan meramente con teorías científicas, sino además responderían a lo que Cuadra-Martínez, Castro y Juliá (2018) denominan teorías subjetivas.

En ambas carreras docentes creen que la argumentación involucra competencias claves para el ejercicio profesional del Derecho y la Filosofía. Mientras en el primer caso esta competencia clave que se centra en la 
capacidad de razonar de acuerdo a un silogismo jurídico y persuadir, en el segundo implicaría el desarrollo de buenas formas de razonar. Esto quiere decir, que mientras en Derecho la argumentación se entiende como un proceso lógico y retórico, en Filosofía se entiende fundamentalmente como un proceso epistémico. Coherente con su inscripción en la formación profesional, estas concepciones acerca de la argumentación son más específicas y precisas que las reportadas por McNeiil et al. (2016) de docentes escolares. Ahora bien, es llamativo que el aspecto deliberativo y dialéctico, clave para el ejercicio de la ciudadanía y el funcionamiento de nuestras democracias (Chambers, 2003) esté completamente ausente de la formación. Si bien se entiende que docentes de ciclos básicos se centren en aspectos distintivos de las profesiones que enseñan, la contribución de estas profesiones a la vida en común debiera ser un foco formativo al que aspirar.

Ahora bien, si bien los y las docentes entrevistados reconocen la importancia de la argumentación, coinciden en que creen que son habilidades que no se enseñan explícitamente en las carreras, lo que es coherente con lo que se reporta en otras carreras universitarias a nivel internacional (Andrews, 2009). Más aún, los y las participantes plantean que no sería parte de su responsabilidad como docentes de Ciclo Básico promover habilidades de argumentación, pues este debería focalizarse en fortalecer conocimiento teórico y habilidades básicas, para promover luego aprender a argumentar. Esto, aun cuando los perfiles intermedios de ambas carreras explicitan la argumentación como una habilidad clave a ser desarrollada en el Ciclo Básico. De esta manera, la creencia de que para argumentar primero hay que manejar rigurosamente el conocimiento, sostendría la idea de que el rol del ciclo básico sería promover el conocimiento para que luego se enseñe a argumentar. Esto es coherente con lo reportado por Andrews (2009) respecto a creencias de docentes de carreras de Biología e Ingeniería, no así de Historia. Considerando que Filosofía y Derecho son carreras humanistas como Historia, este resultado llama la atención. Aún más, dada la evidencia empírica que muestra que el ejercicio de la argumentación fortalece la comprensión de conceptos y teorías (ver Asterhan y Schwarz, 2016), postergar la argumentación para cuando se cuente con conocimiento riguroso parece contraproducente para el mismo objetivo pedagógico buscado, y expresa un desconocimiento del potencial epistémico de la argumentación, como medio de construcción social de conocimiento.

Cuando reportan sus prácticas pedagógicas, aunque la argumentación no aparece como un objetivo de aprendizaje explícito y deliberado, emerge una diversidad de prácticas donde los y las docentes promueven implícitamente habilidades de argumentación. De hecho, se reporta usar estas estrategias para promover aprendizaje activo, participación y comprensión de argumentos filosóficos. Esto es coherente con la evidencia disponible que muestra que estudiantes universitarios avanzan en sus habilidades de argumentación (Larraín et al., 2015; Tuzinkievicz et al., 2018), aun en ausencia de enseñanza explícita. Es decir, aunque los y las participantes plantean que enseñar a argumentar no es el rol del Ciclo Básico, se argumenta para enseñar con poca conciencia de su doble beneficio epistémico. Sin embargo, la mayoría de las interacciones se concentran en clase completa y se desarrolla entre docentes y estudiantes a través de dinámicas de pregunta docente y respuesta de estudiantes, dinámica reportada como crucial en la promoción de argumentación en carreras como Psicología (Bova, 2017). Sin embargo, llama la atención que los intercambios argumentativos aparecen más focalizados en comprender argumentos (foco en Filosofía y Derecho) y construir argumentos persuasivos (foco en Derecho) que en discutir controversias involucrando a pares, lo que efectivamente tendría un mayor efecto en conocimiento (Asterhan y Schwarz, 2016). Esto implica que, aunque las controversias son valoradas y se usan cuando emergen, no se diseñan deliberadamente espacios controversiales, perdiendo un potencial de aprendizaje conceptual relevante. De hecho, el trabajo entre pares aunque aparece, es más circunstancial y no está asociado a un relato respecto a su potencial para la construcción de conocimiento y fortalecimiento de habilidades. Más aún, la enseñanza explícita de la argumentación escrita en el contexto de la construcción de conocimiento disciplinar tampoco es clara, pues no aparece sistemáticamente la existencia de apoyos diseñados para apoyar la escritura argumentativa 0 retroalimentaciones apropiadas. De esta manera, la interacción entre desarrollo de habilidades orales y escritas (Chen et al., 2016) no está explícitamente diseñada. Así, aunque hay una oportunidad importante para desarrollar argumentación, pues se reportan y usarían distintas estrategias muy interesantes que la involucran, falta tener mayor claridad respecto al potencial formativo de la argumentación y estrategias concretas y sistemáticas para ofrecer mejores oportunidades de aprendizaje.

Esto es problemático no solo porque se pierden oportunidades de formación relevantes desde el punto de vista de los objetivos formativos de estas carreras (conocimiento y argumentación), sino porque estas habilidades se asumen y evalúan aunque no se enseñan y no se consideran como responsabilidades formativas. Se observa una invisibilización de la influencia de las habilidades de argumentación en el desempeño de estudiantes (Andrews, 2009), sumado a una dificultad importante para retroalimentar de manera sistemática. Más aún, aparece un discurso que atribuye externamente las dificultades de la enseñanza de manera importante a habilidades básicas y capital cultural de estudiantes, lo que junto con la idea de que las habilidades se desarrollan implícita e individualmente en la familia y estudios de postgrado, podría llevar a asumir las habilidades de argumentación como un dado que los y las estudiantes deberían traer, más que habilidades complejas que se deben enseñar. Esto es coherente con las creencias de docentes 
escolares reportadas por McNeill et al. (2016) para quienes las habilidades de argumentación de los estudiantes tensionan la práctica pedagógica. En el caso de docentes de una Universidad inclusiva en donde se debe resguardar que el desempeño académico no refleje y reproduzca brechas socio-culturales, sino que esté alerta a estas diferencias para compensarlas, la creencia de que las habilidades de argumentación no e sparte de la responsabilidad formativa es de preocupación.

Las creencias reportadas en este estudio no difieren radicalmente de aquellas reportadas para carreras de ingeniería y ciencia en otros países (Andrews, 2009), pero sí de carreras humanistas como historia. Sin embargo, el conocimiento de las creencias de docentes (escolares y universitarios) es incipiente, particularmente en Chile y Latinoamérica. Así, este estudio representa una contribución original y relevante al campo de conocimiento de creencias docentes acerca de la enseñanza de la argumentación, que es reducido. Sugiere que más allá de incluir la argumentación en perfiles de egreso, es necesario discutir su dimensión pedagógica, junto con los supuestos que sostiene el cuerpo docente y diseñar y hacer seguimiento de su formación sistemática.

\section{CONCLUSIONES}

A partir de los resultados se pueden obtener las siguientes conclusiones principales:

1) Docentes de carreras de Filosofía y Derecho no difieren radicalmente en sus creencias acerca del valor de la argumentación para el ejercicio profesional: la argumentación sería una competencia clave para ambas carreras.

2) Coinciden en pensar que la argumentación en tanto competencia se desarrolla principalmente en la práctica y formación de postgrado, creyendo que la responsabilidad formativa de esta competencia recae en años más avanzados de formación, una vez que el conocimeinto teórico esté asentado, aun cuando el perfil intermedio de sus carreras las explicita como competencia clave.

3) Si bien valoran la argumentación, no saben cómo enseñarla y no creen que se enseñe sistemáticamente en sus carreras.

4) Reportan prácticas variadas donde se pone en juego la argumentación oral, centradas en la clase completa y el ejercicio de pregunta docente y respuesta de estudiantes.

5) Existe una invisibilización de la influencia de competencias de argumentación en la evaluación de aprendizaje de sus cursos.

6) Atribuyen las dificultades de enseñanza principalmente a la carencia de habilidades básicas de los y las estudiantes.

\section{MATERIAL SUPLEMENTARIO}

El guión temático de la entrevista, los perfiles intermedios y de egreso y las estrategias de enseñanza declaradas están disponibles para ser solicitados a Leandro De Brasi (Idebrasi@uahurtado.cl).

\section{AGRADECIMIENTOS}

Los autores agradecemos el apoyo financiero del programa PMI de la Universidad Alberto Hurtado a través del proyecto UAH1501 y del programa FONDECYT de ANID a través del proyecto \#1180886. Agradecemos también la colaboración investigativa de Macarena Sanhueza Céspedes.

\section{REFERENCIAS}

Andrews, R., Argumentation in higher education. Improving practice through theory and research. Routledge, New York, USA (2009).

Asterhan, C. S., y Schwarz, B. B., Argumentation for learning: Well-trodden paths and unexplored territories, https://doi.org/10.1080/00461520.2016.1155458, Educational Psychologist, 51(2), 164-187 (2016).

Blasi, V., Teaching Reasoning. Chicago-Kent Law Review, 74(2), 647-654 (1999).

Bova, A., The Role of the Teacher in Promoting Argumentative Interactions in the Learning Contexts of Higher Education; en F. Arcidiacono y A. Bova. Interpersonal Argumentation in Educational and Professional Contexts, pp 75-94. Springer International Publishing. (2017).

Chambers, S., Deliberative Democratic Theory, Annual Review of Political Science, 6, 307-26. (2003). 
Chen, Y.Ch., B., y Soonhye, P., Examining elementary students' development of oral and written argumentation practices through argument-based inquiry, http://dx.doi.org/10.1007/s11191-016-9811-0, Science \& Education 25(3-4), 277-320 (2016).

Cuadra-Martínez, D. J., Castro, P. J., y Juliá, M. T., Tres saberes en la formación profesional por competencias: integración de teorías subjetivas, profesionales y científicas, http://dx.doi.org/10.4067/S0718-50062018000500019, Formación Universitaria, 11(5), 19-30 (2018).

Coirier, P., Andriessen, J y Chanquoy, L.. From Planning to Translating: The Specificity of Argumentative Writing; en Andriessen, J. y Coirier, P. Foundations of Argumentative Text Processing, pp 1-28. Amsterdam: Amsterdam University Press. (1999).

Felton, M., The development of discourse strategies in adolescent argumentation, https://doi.org/10.1016/j.cogdev.2003.09.001, Cognition \& Development, 19(1), 35-52. (2004).

Felton, M., y Kuhn, D., The development of argumentative discourse skill, https://doi.org/10.1080/0163853X.2001.9651595, Discourse Process, 32(2-3), 135-153. (2001).

Greco, S., Using argumentative tools to understand inner dialogue, Argumentation, 31(2), 331-358. (2017).

Habermas, J., Between facts and norms. Contributions to a discourse theory of law and democracy. MIT Press, Massachusetts, USA (1996).

Harrell, M., Argument diagramming and critical thinking in introductory philosophy, https://doi.org/10.1080/07294360.2010.502559, Higher Education Research \& Development, 30(3), $371-385$ (2011).

Iordanou, K., Kuhn, D., y otros tres autores, Learning by arguing, Learning and Instructrion, 63, 101207 (2019).

Iliadi, S., Theologou, K., y Stelios, S., Are University Students Who Are Taking Philosophy Courses Familiar with the Basic Tools for Argument? https://doi.org/10.5840/teachphil2019726106, Teaching Philosophy, 42, 197-220 (2019).

Kuhn, D., Critical thinking as discourse, https://doi.org/10.1159/000500171, Human Development, 62(3), 146-164 (2019).

Larraín, A. y Burrows, F., Las pautas sí importan. Efecto del uso de pautas sobre la calidad de escritura argumentativa en la universidad, https://doi.org/10.4067/S0718-50062020000100115, Formación Universitaria, 13(1), 115-126. (2020).

Larraín, A., Freire, P., y otros tres autores, ¿La Universidad de Chile promueve las habilidades de argumentación escrita: Un estudio exploratorio comparativo de estudiantes de educación universitaria y educación técnica, Calidad en la Educación, 43, 201-228. (2015).

Larraín, A., Freire, P., y Howe, C., Science teaching and argumentation: One-sided versus dialectical argumentation in Chilean middle school science lessons, https//doi.org/10.1080/09500693.2013.832005, International Journal of Science Education, 36(6), 1017-36 (2014).

Leitão, S., The Potential of Argument of knowledge Building, Human Development, 43, 332-360 (2000).

Martin, S., y Dismuke, S., Investigating differences in teacher practices through a complexity theory lens: The influence of teacher education, https://doi.org/10.1177/0022487117702573, Journal of Teacher Education, 69(1), 22-39, (2018)

Martinich, A.P., Philosophical Writing, 4ª edición. John Wiley \& Sons, London, UK (2016).

Mcnaughton, S., Zhu, T., y otros cuatro autores, Critical perspective taking: promoting and assessing online written argumentation for dialogic focus, Studia Paedagogica, 24(4), 119-141 (2019).

McNeill, K., Katsh-Singer, R., y otros dos autores, Factors impacting teachers' argumentation instruction in their science classrooms, https://doi.org/10.1080/09500693.2016.1221547, International Journal of Science Education, 38(12), 20262046 (2016).

Newell, G.E., Beach, R., y otros dos autores, Teaching and Learning Argumentative Reading and Writing: A Review of Research, https://doi.org/10.1598/RRQ.46.3.4, Reading Research Quarterly, 46(3), 273-304 (2011).

Pinkwart, N., Aleven, V., Ashley, K., y Lynch, C., Using Collaborative Filtering in an Intelligent Tutoring System for Legal Argumentation. Proceedings of Workshops held at the 4th International Conference on Adaptive Hypermedia and Adaptive Web-Based Systems, pp 542-51. National College of Dublin, Dublin, Ireland. (2006).

Rapanta, C., Garcia-Mila, M., y Gilabert, S., What is meant by argumentative competence? An integrative review of methods of analysis and assessment in education, https://doi.org/10.3102/0034654313487606, Review of Educational Research, 83(4), 483-520 (2013).

Robbins-Tiscione, K.K., Philosophy v. Rhetoric in Legal Education: Understanding the Schism Between Doctrinal and Legal Writing Faculty, Journal of the Association of Legal Writing Directors, 3, 108-128 (2006).

Stein, N. L., y Albro, E. R., The origins and nature of arguments: Studies in conflict understanding, emotion, and negotiation. Discourse processes, 32(2-3), 113-133 (2001).

Tuzinkievicz, M.A., Peralta, N.S., y otros dos autores, Complejidad argumentativa individual escrita en estudiantes universitarios ingresantes y avanzados. LIBERABIT. Revista Peruana de Psicología, 24(2), 231-247 (2018). 